\title{
Discrete Phase Modeling of Nanofluid Flow around a Circular Bluff Body
}

\author{
R. Deepak Selvakumar, S. Dhinakaran \\ The Centre for Fluid Dynamics, Department of Mechanical Engineering, Indian Institute of Technology Indore \\ Khandwa Road, Simrol, Indore 453 552, Madhya Pradesh, India \\ phd1301203004@iiti.ac.in; sdhina@iiti.ac.in
}

\section{Extended Abstract}

Forced convection heat transfer around a circular bluff body is a scenario that occurs in many engineering applications such as electronic cooling, nuclear reactors, heat exchangers and so on. This classic area of research has gained much more momentum with the advent of nanofluids which are promising alternatives to conventional coolants due to their superior thermal properties. Many numerical studies on nanofluid flow and heat transfer using Single-Phase Modeling (SPM) and Multi-Phase Modeling (MPM) with Eulerian-Eulerian approach are available in literature. However, numerical studies using Discrete Phase Modeling (DPM) with Eulerian-Lagrangian approach which has the advantage of tracking individual particles and accounting for the interactions and momentum transfer between the fluid and particles are very limited [1].

In this study, a steady, laminar and forced convective nanofluid flow around a 2-D circular cylinder has been numerically analyzed using Discrete Phase Modeling (DPM). Steady state governing equations of flow and heat transfer for the basefluid and the equation of nanoparticle motion were solved using a Finite Volume Method (FVM) and trajectory analysis approach, respectively. An Eulerian approach was employed for the basefluid, while a Lagrangian approach was used for calculating the particle trajectory with two-way coupling. The continuous phase (basefluid $-\mathrm{H}_{2} \mathrm{O}$ ) was considered to carry the discrete phase (nanoparticle $-\mathrm{Al}_{2} \mathrm{O}_{3}$ ) and the momentum transfer between the nanoparticles and basefluid was also taken into account. The nanoparticles were assumed to be randomly dispersed in the continuous phase and the influences of Brownian motion, thermophoresis, drag and nanoparticle weight were also considered.

The flow and heat transfer was analyzed at $10 \leq R e \leq 40$ and nanoparticle volume fraction varying from $0 \%$ to $5 \%$. In general, the heat transfer increased with the addition of nanoparticles and the augmentation in heat transfer was more pronounced at higher Reynolds numbers. The influence of reflect and trap boundary conditions over the cylinder was also analyzed. The results of DPM were compared with that of the SPM approach. The results of DPM showed that the nanoparticle concentration is not uniform, which is contradictory with the basic assumption of SPM. In fact, a drop in concentration near the walls was observed for trap boundary condition whereas, a slight increase in concentration was noted in reflect boundary condition. Furthermore, addition of nanoparticles resulted in a marginal increase in total drag coefficient. Brownian motion exhibited more influence on the heat transfer augmentation than thermophoresis. It is believed that the Discrete Phase Modeling (DPM) using Eulerian-Lagrangian approach with two-way coupling is more realistic than other two approaches. But, it is also necessary to mention that the SPM and MPM are simpler and computationally less expensive than DPM.

\section{References}

[1] N. A. C. Sidik, M. N. A. W. M Yazid, S. Samion, M .N. Musa, R. Mamat, "Latest development on computational approaches for nanofluid flow modeling: Navier-Stokes based multiphase models," Int. Commun. Heat. Mass., vol. 74, pp. 114-124, 2016. 